OPEN ACCESS

Edited by:

Jens Geginat,

Istituto Nazionale Genetica

Molecolare (INGM), Italy

Reviewed by:

Markus Kleinewietfeld,

VIB-UGent Center for Inflammation

Research, Belgium

Maria Cecilia Giron,

Università degli Studi di

Padova, Italy

*Correspondence:

Mario Clerici

mario.clerici@unimi.it,

mclerici@dongnocchi.it

tThese authors have contributed equally to this work.

Specialty section:

This article was submitted

to Multiple Sclerosis and

Neuroimmunology,

a section of the journal

Frontiers in Immunology

Received: 22 June 2017 Accepted: 09 October 2017 Published: 25 October 2017

Citation:

Saresella M, Mendozzi L, Rossi V, Mazzali F, Piancone F, LaRosa F,

Marventano I, Caputo D, Felis GE and Clerici M (2017) Immunological and Clinical Effect

of Diet Modulation of the Gut Microbiome in Multiple Sclerosis

Patients: A Pilot Study.

Front. Immunol. 8:1391. doi: 10.3389/fimmu.2017.01391

\section{Immunological and Clinical Effect of Diet Modulation of the Gut Microbiome in Multiple Sclerosis Patients: A Pilot Study}

\author{
Marina Saresella ${ }^{1 \dagger}$, Laura Mendozzi ${ }^{2 t}$, Valentina Rossi ${ }^{2}$, Franca Mazzali ${ }^{2}$, \\ Federica Piancone', Francesca LaRosa', Ivana Marventano', Domenico Caputo'2, \\ Giovanna E. Felis ${ }^{3}$ and Mario Clerici, ${ }^{1,4 *}$
}

'Laboratory of Molecular Medicine and Biotechnology, Don Gnocchi Foundation, IRCCS, Milan, Italy, ${ }^{2}$ Department of Neurology, Don Gnocchi Foundation, IRCCS, Milan, Italy, ${ }^{3}$ Department of Biotechnology, University of Verona, Verona, Italy, ${ }^{4}$ Department of Physiopathology and Transplants, University of Milano, Milan, Italy

Pathogenesis of autoimmune disorders, including multiple sclerosis (MS), has been linked to an alteration of the resident microbial commensal community and of the interplay between the microbiota and the immune system. Dietary components such as fiber, acting on microbiota composition, could, in principle, result in immune modulation and, thus, could be used to obtain beneficial outcomes for patients. We verified this hypothesis in a pilot study involving two groups of clinically similar relapsing-remitting (RR) MS patients who had undergone either a high-vegetable/low-protein diet (HV/LP diet group; $N=10$ ) or a "Western Diet" (WD group; $N=10$ ) for at least 12 months. Gut microbiota composition, analyzed by $16 \mathrm{~S}$ V4 rRNA gene sequencing and immunological profiles, was examined after a minimum of 12 months of diet. Results showed that, in the HV/LP diet group compared to the WD group: (1) Lachnospiraceae family was significantly more abundant; (2) IL-17-producing T CD4+ lymphocytes $(p=0.04)$ and PD-1 expressing T CD4+ lymphocytes $(p=0.0004)$ were significantly decreased; and (3) PD-L1 expressing monocytes $(p=0.009)$ were significantly increased. In the HV/LP diet group, positive correlations between Lachnospiraceae and both CD14+/ $\mathrm{IL}-10+$ and CD14+/TGF $\beta+$ monocytes $\left(R_{\mathrm{Sp}}=0.707, p=0.05\right.$, and $R_{\mathrm{Sp}}=0.73$, $p=0.04$, respectively), as well as between Lachnospiraceae and CD4+/CD25+/ FoxP3+ T lymphocytes $\left(R_{\mathrm{Sp}}=0.68, p=0.02\right)$ were observed. Evaluation of clinical parameters showed that in the HV/LP diet group alone the relapse rate during the 12 months follow-up period and the Expanded Disability Status Scale score at the end of the study period were significantly reduced. Diet modulates dysbiosis and improves clinical parameters in MS patients by increasing anti-inflammatory circuits. Because Lachnospiraceae favor Treg differentiation as well as TGF $\beta$ and IL-10 production this effect could be associated with an increase of these bacteria in the microbiota.

\section{Keywords: multiple sclerosis, diet, microbiome, cytokine, inflammation, dysbiosis, immunology}

Abbreviations: MS, multiple sclerosis; CNS, central nervous system; EDSS, Expanded Disability Status Scale; IL, interleukin; OTU, operation taxonomic units; WD, Western Diet; HV/LP, high vegetal/low-protein DIET. 


\section{INTRODUCTION}

Multiple sclerosis (MS) is a chronic disease of the central nervous system (CNS) characterized by demyelination and mediated by an auto-reactive immune process directed against central neural tissues. Experimental autoimmune encephalitis (EAE) is a widely used animal model of MS induced by CNSrestricted antigens (1). The ethiopathogenesis of MS is still only partly understood, but a number of recent publications suggested that alterations of the microbiota play a role in the pathogenesis of this disease (2-4). Thus, the use of a cocktail of antibiotics to alter the gut microbiota of mice prior to EAE induction was shown to result in a significant reduction of EAE severity. This effect was mediated by an increase of $\mathrm{CD} 4+1$ CD25+/FoxP3+ regulatory T cells (Treg) cells (5) and of regulatory CD5+/B cells (6). Even more recently, results indicated that EAE-resistant germ-free mice are rendered susceptible to the disease by the introduction of segmented filamentous bacteria into their gut microbiome. This phenomenon was the consequence of an increased differentiation of proinflammatory Th17 cells (7). Notably, dietary supplementation with probiotics was shown to modulate EAE secondarily to the regulation of pro- and anti-inflammatory cytokines (8-16), and engineered bacteria strains, such as Salmonella-CFA/I and Hsp65-producing Lactococcus lactis, were observed to prevent EAE via the production of TGF $\beta$ and IL-13 by Tregs (17-19). Finally, diet has been observed to influence EAE susceptibility and disease activity (2). Thus, a low-calorie diet was shown to have a beneficial effect in EAE (20), whereas a salt-rich diet resulted in an increased severity of EAE as a consequence of an upregulation of Th17 cell activity (21).

The analysis of gut microbiota in MS patients is still in the early stages (2). Recent data (22) showed that the human gut is colonized by Clostridium perfringens type B during disease relapse. Clostridium perfringens type $B$ produces a toxin ( $\varepsilon$ toxin) that causes microangiopathy, resulting in the disruption of the blood-brain barrier (BBB) associated with neuronal and oligodendrocyte damage (23-26), possibly justifying its effect of disease activity. Additional data indicated that a mixture of Clostridium species enhances Treg cell populations in MS patients (27), suggesting that, besides the effect of a toxin, an imbalance within Clostridium species (phylum Firmicutes) might be present in the microbiota of these patients. Moreover, the archaeal Methanobrevibacteriaceae was described to be increased in MS patients, in whom the anti-inflammatory taxa Butyricimonas (phylum Bacteroidetes) and Lachnospiraceae (phylum Firmicutes) were decreased (28). Even more recently, Faecalibacterium prausnitzii, an important butyrate-producing organism, was observed to be reduced in MS patients in analogy to what is observed in inflammatory bowel disease, another autoimmune condition (29). Since butyrate upregulates Treg cell populations, these results suggest a mechanism by which gut microbiome alterations would predispose individuals to developing MS.

As diet plays an essential role in shaping the gut microbiome (30), and a high-fiber intake has been linked to health benefits as a consequence of the effect of fiber on the gut microbiota (31), possibly resulting in the modulation of the immune response, we hypothesized that MS disease activity could be affected by dietary patterns. In this pilot study, we verified this hypothesis by analyzing immune indexes, clinical parameters and gut microbiota in two groups of MS patients who at the time of recruitment were already following two distinct dietary regimes: a "Western Diet" (WD) and a high-vegetable/low-protein diet (HV/LP diet).

\section{MATERIALS AND METHODS}

\section{Individuals Enrolled in the Study}

Patients with a diagnosis of relapsing-remitting (RR) MS that are followed by the Multiple Sclerosis Rehabilitation Unit of the Don Carlo Gnocchi Foundation in Milan, Italy, were enrolled in this pilot trial on a voluntary basis between May and October of 2016. Notably, this unit offers dietary advices to patients that are provided by a staff of professional nutritionists. Inclusion criteria were age $>18$ years and disease stability for $>6$ months prior to enrollment. Main exclusion criteria were: (1) use of disease modifying treatment (DMD) for $>6$ months prior to enrollment; (2) use of immunosuppressants or teriflunomide in the clinical history; (3) presence of significant co-morbidities such as arterial hypertension, cerebrovascular disorders, heart or pulmonary diseases, diabetes, endocrine, gastrointestinal, or psychiatric diseases. Gender, disease duration, and disability level, as assessed by the Kurtzke Expanded Disability Status Scale score (EDSS), relapse rate and other neurological indices were not used as inclusion/exclusion criteria but were recorded during the initial neurological examination.

Twenty-nine patients were initially selected for the study; all the patients underwent a face-to-face interview with a team of professional nutritionists who assessed the dietary regimen that had been followed for at least a 12-months period. This period was selected because it was considered to be a valid way to assess the adoption of a stable dietary habit. Nine of the initially selected patients were not enrolled in the study because adherence to clear dietary patterns could not be unequivocally identified. Of the remaining 20 patients, 10 (7 females and 3 males; median age $=43, \mathrm{IQ}=40-44)$ had chosen to follow a diet characterized by the use of fresh fruits and vegetables, legumes, nuts, whole grains, and extra virgin olive oil and a very limited use of animal proteins, including fish (no more than twice a week), poultry (no more than once a week), eggs (no more than four eggs a week), and dairy products (no more than once a week), as well as a low intake of refined cereals, salt, sugar, fried food and the exclusion of alcohol, red meat, saturated fats of animal origin, and trans-fats (e.g., processed dressing). This diet was labeled as high vegetable/ low protein (HV/LP diet). The remaining 10 patients ( 8 females and 2 males; median age $=49, \mathrm{IQ}=45-52$ ) were following a classical Western Diet (WD) characterized by the regular consumption of red meat, processed meat, refined grains, sweetened food, salt, and an overall high intake of saturated and omega- 6 fatty acids (32). These 20 patients were enrolled in the study. Adherence to the two different dietary regimens was verified in a face-to-face interview with the professional nutritionists every 4 months. No use of any type of antibiotic or of pre- and probiotics was reported 
during the study period. Blood and fecal samples were collected at enrollment, i.e., after at least 1 year of either WD or HV/LP diet; neurological examinations were performed at enrollment and at the 12 months follow-up point.

The study protocol was approved by the ethics committee of the Don Carlo Gnocchi Foundation and all the enrolled patients signed an informed consent.

\section{Blood Sample Collection and Cell Separation}

At enrollment whole blood $(10 \mathrm{ml})$ was collected in vacutainer tubes containing ethylenediaminetetraacetic acid (EDTA) (Becton Dickinson \& Co., Rutherford, NJ, USA). Peripheral blood mononuclear cells (PBMC) were separated on lympholyte separation medium (Cedarlane, Hornby, Ontario, CA, USA) and washed twice in PBS at 1500 RPM for 10 min; viable leukocytes were determined using a Scepter 2.0 Handheld Automated Cell Counter (Millipore, Billerica, MA, USA).

\section{Intracellular Cytokine or Transcription Factor Staining in PBMC}

Lymphocyte and monocyte subsets were analyzed in freshly isolated PBMC that were incubated for $30 \mathrm{~min}$ at $4^{\circ} \mathrm{C}$ in the dark with Phycoerythrin-Cyanin-7 (PC7)-labeled anti-CD4 (clone

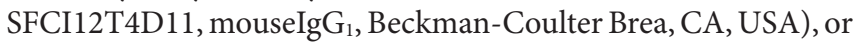
PC7-labeled anti-CD14 (clone RMO52, mouse IgG 2a $_{2}$, BeckmanCoulter), Phycoerythrin-Texas Red (ECD)-labeled anti-CD25 (clone B1.49.9, mouse IgG Iga $_{2}$, Beckman-Coulter), Phycoerythrin (PE)-labeled anti-PD-1 (clone MIH4, mouse IgG1, eBioscience Cornerstone Court West, San Diego, CA, USA), PE-labeled antiPD-L1 (clone MIH1, mouse IgG1, eBioscience), or PE-labeled anti-human Tim-3 (clone 344823, rat IgG IfA $_{2}$ R\&D Systems, Inc., Minneapolis, MN, USA). After incubation, the cells were washed, treated with Cell Permeabilization kit (FIX \& PERM kit, eBioscience) and incubated for $30 \mathrm{~min}$ at $4^{\circ} \mathrm{C}$ in the dark with the following PE-labeled monoclonal antibodies: anti-IL-10 (clone JES9D7, mouse IgG1, R\&D Systems), anti-TGF $\beta$ (clone 9016, mouse IgG1, R\&D Systems), anti-IFN $\gamma$ (clone 25723, mouse IgG Ib $_{\text {b }}$ R\&D Systems), anti-BDNF (clone 35909, mouse- IgG1, R\&D Systems), anti-IL-25 (IL-17E, clone 182203, mouse IgG1, R\&D Systems), anti-Gal-9 (clone 9M1-3, mouse IgG1 $1_{k}$, Biolegend, San Diego, CA, USA), anti-RORC $\gamma$ (clone AFKJS-9, rat IgG2a, eBioscience), anti-GATA-3 (cloneTWAY, rat IgG2B, eBioscience), anti-NFATc1

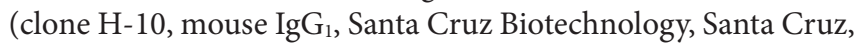
CA, USA), or the Fluorescein Isothiocyanate (FITC)-labeledanti-NFkB (clone C-5, mouse $\operatorname{IgG}_{2 \mathrm{a}}$, Santa Cruz Biotechnology), the PC-5-labeled-anti-IL-17 (clone BL168, mouse IgG I $_{1 \mathrm{k}}$, Biolegend), or the Alexa Fluor 488-labeled-anti-FoxP3 (clone $1054 \mathrm{C}$, rabbit IgG, R\&D). Anti-Bat3 (clone 2B21, mouse $\operatorname{IgM}_{\mathrm{k}}$, Abnova Taipei, Taiwan) was conjugated using the LightningLinkTM R-Phycoerythrin conjugation kit (Innova Biosciences, Cambridge, UK).

\section{Flow-Cytometry Analysis}

Peripheral blood mononuclear cells were analyzed using a Beckman-Coulter GALLIOS flow cytometer equipped with a
$22 \mathrm{~mW}$ Blue Solid State Diode laser operating at $488 \mathrm{~nm}$ and with a $25 \mathrm{~mW}$ Red Solid State Diode laser operating at $638 \mathrm{~nm}$, and interfaced with Kaluza analysis software. Two hundred thousand cells were acquired and gated on lymphocyte and monocyte FSC and SSC properties. Isotype control or single fluorochromestained preparations were used for color compensation.

\section{Microbiome Analyses}

At enrollment, participants were asked to collect their first morning stool at home using an adequate stool collection container (Biosigma, VE, ITALY). Samples were shipped, within $1 \mathrm{~h}$, on ice packs to the Laboratory of the Don Carlo Gnocchi Foundation in Milan, where they were immediately stored at $-80^{\circ} \mathrm{C}$. Finally, the stored total stool samples were sent on dry ice by FedEx delivery to the processing facility (Second Genome Inc., San Francisco, CA, USA).

DNA isolation, library preparation, and sequencing as well as data analysis were performed by Second Genome Inc. Briefly, nucleic acid isolation with the MoBio PowerMag ${ }^{\circledR}$ Microbiome kit (Carlsbad, CA, USA) and quantified via the Qubit ${ }^{\circledR}$ Quant-iT dsDNA High Sensitivity Kit (Invitrogen, Life Technologies, Grand Island, NY). Samples enriched in bacterial $16 \mathrm{~S}$ V4 rDNA region and incorporating Illumina (San Diego, CA, USA) adapters and indexing barcodes, by PCR, were concentrated using a solidphase reversible immobilization method for the purification of PCR products, quantified by qPCR and sequenced with MiSeq ${ }^{\circledR}$ instrument. Amplicons were sequenced for 250 cycles with custom primers designed for paired-end sequencing. Operation taxonomic units (OTU) were selected using an in-house pipeline of analysis and sequences hitting a unique strain with an identity $\geq 99 \%$ were assigned a strain OTU. To ensure specificity of the strain hits, a difference of $>=0.25 \%$ between the identity of the best hit and the second best hit was required (e.g., 99.75 vs. 99.5); a chimera filtering and discard was also used. Representative OTU sequences were assigned taxonomic classification via mothur's Bayesian classifier, trained against the Greengenes reference database of $16 \mathrm{~S}$ rRNA gene sequences clustered at 99\%.

As for alpha-diversity (within sample diversity), observed diversity (number of unique OTU) and Shannon Index (which utilizes the richness of a sample along with the relative abundance of the present OTUs to calculate a diversity index) were the metrics used.

Sample-to-sample dissimilarity (beta diversity) was also determined. All profiles are inter-compared in a pair-wise fashion to determine a dissimilarity score and store it in a distance dissimilarity matrix. Distance functions produce low dissimilarity scores when comparing similar samples. Abundance-weighted sample pair-wise differences were calculated using the Bray-Curtis dissimilarity (ratio of the summed absolute differences in counts to the sum of abundances in the two samples) (33). The binary dissimilarity values were calculated with the Jaccard index (metric comparing the number of mismatches, i.e., OTUs present in one but absent in the other, in two samples relative to the number of OTUs present in at least one of the samples) (34).

Whole Microbiome Significance Testing was performed with Permutational Analysis of Variance (PERMANOVA), utilized for finding significant differences among discrete categorical or 
continuous variables. To identify differentially abundant taxa, a Wilcoxon Rank Sum test was employed. $p$ values were adjusted by Benjamini-Hochberg procedure to control for false discovery rates from multiple testing. For additional information on laboratory methods and bioinformatic analyses please see Data Sheet S1 in Supplementary Material.

\section{Statistical Analysis}

Quantitative data were not normally distributed (Shapiro-Wilk test) and are, thus, summarized as median and interquartile range (IQR; $25^{\circ}$ and $75^{\circ}$ percentile). Comparisons between two MS groups were made using a two-tailed Mann-Whitney $U$ test performed for independent samples. The statistical correlations between immunological parameters and microbiota data were investigated by means of Spearman correlation coefficient and 95\% confidence limits performed by Fisher's $Z$ transformation. The top 8 most abundant taxonomic families were compared by Kruskal-Wallis (KW) rank sum test. $\mathrm{x}^{2}$ test was used to patients relapse comparison. Statistical significance was set at a $p$-value $<0.05$. Data analysis was performed using the MedCalc statistical package (MedCalc Software bvba, Mariakerke, Belgium).

\section{RESULTS}

\section{Clinical Characteristics of the Individuals Enrolled in the Study}

Demographic and clinical characteristics of the individuals enrolled in the study are summarized in Table 1. No differences were observed in gender, age, disease duration relapse rate, and EDSS score status when the two groups were compared at enrollment.

\section{Diet-Associated Modifications of the Microbiota}

Microbiota analyses were performed in all the MS individuals included in this study at enrollment, i.e., after at least 1 year of either HV/LP diet or WD. Sequences per sample ranged

TABLE 1 | Demographic and clinical characteristics of patients with a diagnosis of multiple sclerosis who were following either a Western Diet (WD) or a highvegetable/low-protein (HV/LP) diet.

\begin{tabular}{lccc}
\hline A & WD & HV/LP diet & p-Value \\
\hline Number & 10 & 10 & \\
Gender (M:F) & $2: 8$ & $3: 7$ & \\
Age years (range years) & $49(45-52)$ & $43(40-44)$ & 0.1 \\
$\begin{array}{l}\text { Disease duration years } \\
\text { (range years) }\end{array}$ & $12.5(4.3-17.8)$ & $8.8(4-15)$ & 0.4 \\
$\begin{array}{l}\text { Expanded Disability Status } \\
\begin{array}{l}\text { Scale (range) } \\
\text { Relapse rate (relapse number/ }\end{array}\end{array}$ & $2.0(1.6-1.9)$ & $1.8(1.3-2.0)$ & 0.3 \\
disease years) & $0.3(0.3-0.8)$ & $1(0.0-1.0)$ & 0.4 \\
\hline
\end{tabular}

Results obtained at enrollment (i.e., after 1 year of either WD or HVILP diet) are shown. Data are reported as medians and interquartile range. Statistical significance is presented $(p<0.05)$. between 138,072 and 405,385 filtered reads and were sequenced to sufficient depth to capture OTU richness (Image 1). As a whole, 1,550 OTUs (combined filtered and strain level hits) were obtained from 8,852,375 combined sequences. All filtered reads were classified at the Kingdom level, $93.08 \%$ of reads were classified at the family level, $54.78 \%$ of reads were classified at the genus level, $22.7 \%$ of reads were classified at the species level and $23.87 \%$ of reads were classified at the strain level.

Firmicutes was the most abundant phylum (Table 2; Figure 1) and Ruminococcaceae and Lachnospiraceae were the most abundant families in both groups of patients (Table 3; Figure 2). There was no difference in alpha-diversity when individuals following either one of the diets were compared (Figure 3), while Lachnospiraceae was significantly more abundant in the patients following HV/LP diet (Table 3) and phylum Euryarchaeota was significantly more abundant in WD patients $(p=0.03)$ (Table 2). No significantly different abundant OTUs between the two diets were observed at the time of sampling, even though 66 OTUs had an unadjusted $p$-value $<0.05$ and absolute log 2 -fold change greater than 1 (data not shown). Moreover the taxa, namely Coprococcus eutactus $(p=0.3)$, Ruminococcus lactaris $(p=0.03)$ and a sequence of an as-yet unclassified Lachnospiraceae strain $(p=0.03)$, Roseburia intestinalis $(p=0.03)$, and a Hungatellarelated unknown Lachnospiraceae member $(p=0.04)$ appeared to be more abundant in HV/LP (Table S1 in Supplementary Material).

Notably, although being suggestive of a role for the different dietary regimens in the changes in the microbiota composition, as baseline samples were not collected, it is not possible to definitely state that such changes are the direct consequence of the dietary regimens.

\section{Immune Parameters}

Immune parameters were analyzed in all the individuals at enrollment, i.e., after at least 1 year of either HV/LP diet or WD. Results showed that three cell populations were significantly different when HV/LP diet and WD were compared. Thus, in HV/LP diet compared to WD: (1) IL-17+/CD4+ as well as CD4+/PD-1+
TABLE 2 | Median percent relative abundance and interquartile range of the most abundant taxa at the phylum level in patients with a diagnosis of multiple sclerosis who were following either a Western Diet (WD) or a high-vegetable/lowprotein (HV/LP) diet.

\begin{tabular}{lcccc}
\hline Phylum & WD & HV/LP diet & $\boldsymbol{p}$-Value & Chi-square \\
\hline Firmicutes & $76.8(68.8-81.9)$ & $73.5(70.7-79.3)$ & 0.9 & 2.7 \\
Bacteroidetes & $10.8(8.9-13.7)$ & $13.6(11.8-17.6)$ & 0.2 & 1.5 \\
Actinobacteria & $8.1(3.6-10.2)$ & $5.7(1.6-9.9)$ & 0.5 & 0.4 \\
Proteobacteria & $1.4(0.5-3.0)$ & $1.3(0.4-2.3)$ & 0.5 & 0.3 \\
Verrucomicrobia & $0.0(0.0-0.04)$ & $0.0(0.0-0.3)$ & 0.6 & 0.1 \\
Euryarchaeota & $0.04(0.0-0.8)$ & $0.0(0.0-0.05)$ & $\mathbf{0 . 0 3}$ & 4.3 \\
Tenericutes & $0.0(0.0-0.4)$ & $0.0(0.0-0.5)$ & 0.8 & 0.04 \\
unclassified & $0.1(0.0-0.1)$ & $0.05(0.04-0.06)$ & 0.8 & 0.03 \\
Others & $0.0(0.0-0.1)$ & $0.01(0.0-0.03)$ & 0.4 & 0.4 \\
\hline
\end{tabular}

Results obtained at enrollment (i.e., after 1 year of either WD or HV/LP diet) are shown. Statistical significance is presented $(p<0.05)$.

The bold values are statistically significant $p$ values. 


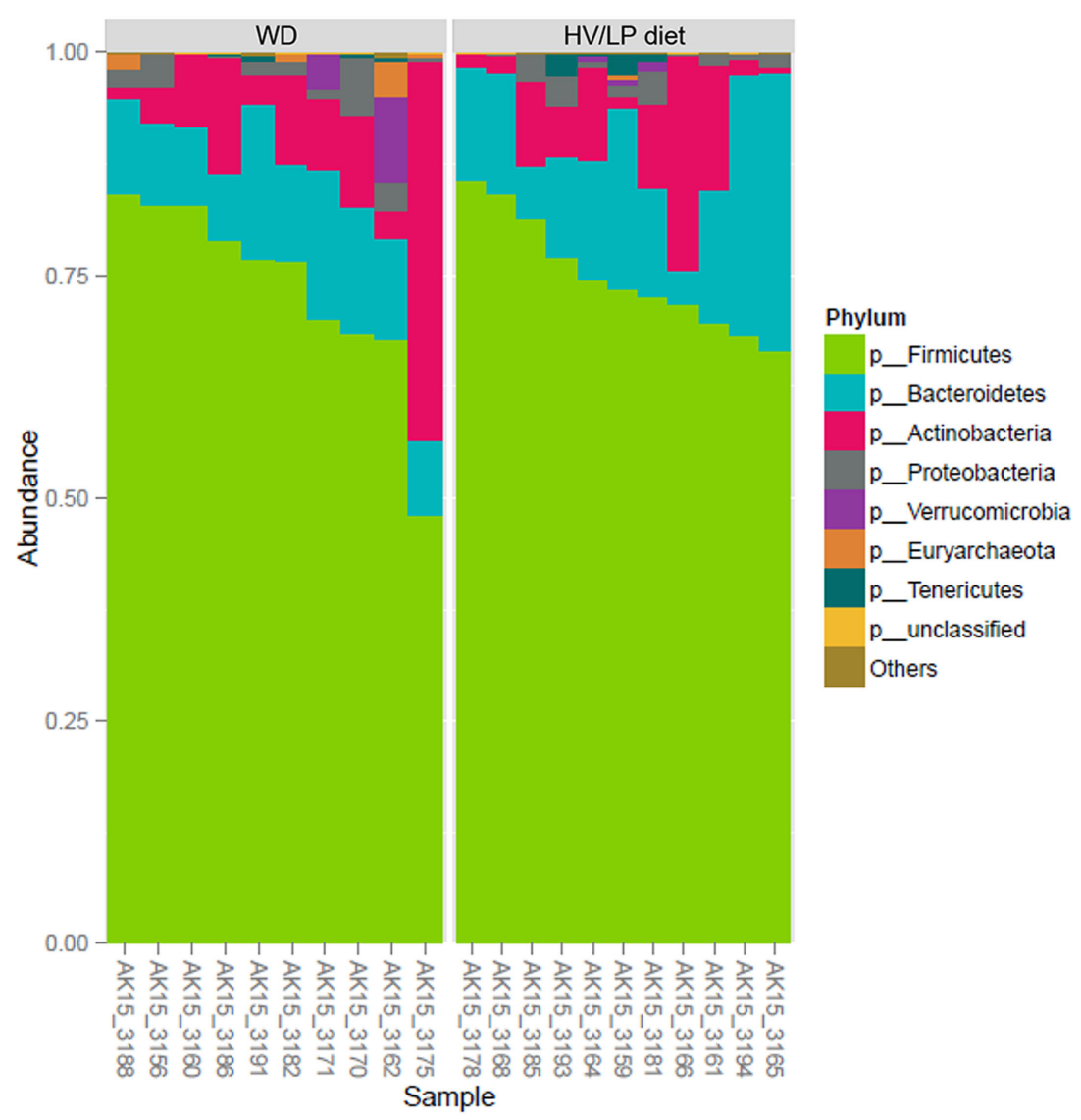

FIGURE 1 | Firmicutes was the most abundant phylum in both groups (about 75\%), followed by Bacteroidetes (15\%), Actinobacteria (10\%), and Proteobacteria $(<2 \%)$. Plot showing the most abundant taxa at the Phylum level in multiple sclerosis (MS) patients who were following either a Western Diet (WD) or a highvegetable/low-protein (HV/LP) diet. Stool samples were collected at enrollment, i.e., after at least 1 year of either WD or HV/LP diet.

TABLE 3 | Median percent relative abundance and interquartile range of the most abundant taxa at the family level in patients with a diagnosis of multiple sclerosis who were following either a Western Diet (WD) or a high-vegetable/low-protein (HV/LP) diet.

\begin{tabular}{lcccc}
\hline Family & WD & HV/LP diet & $\boldsymbol{p}$-Value & Chi-square \\
\hline Ruminococcaceae & $31.5(24.7-35.7)$ & $29.7(27.1-33.6)$ & 0.83 & 0.04 \\
Lachnospiraceae & $21.8(20.0-24.9)$ & $29.5(24.4-30.9)$ & $\mathbf{0 . 0 4}$ & $\mathbf{4 . 1}$ \\
Bacteroidaceae & $6.0(5.1-8.1)$ & $7.7(6.2-9.7)$ & 0.36 & 0.8 \\
Bifidobacteriaceae & $6.2(1.7-8.1)$ & $3.5(0.7-9.1)$ & 0.67 & 0.1 \\
Erysipelotrichaceae & $4.8(3.3-9.9)$ & $1.6(1.3-6.9)$ & 0.32 & 0.9 \\
Veillonellaceae & $0.9(0.5-4.3)$ & $1.6(0.6-2.2)$ & 0.94 & 0.005 \\
Coriobacteriaceae & $1.9(1.5-2.3)$ & $1.0(0.6-1.9)$ & 0.11 & 2.6 \\
Prevotellaceae & $0.9(0.0-1.4)$ & $0.0(0.0-0.1)$ & 0.29 & 1.1 \\
Others & $16.6(13.8-20.7)$ & $16.1(13.3-18.6)$ & 0.48 & 0.4 \\
\hline
\end{tabular}

Results obtained at enrollment (i.e., after 1 year of either WD or HV/LP diet) are shown. Statistical significance is presented $(p<0.05)$.

The bold values are statistically significant $p$ values.

T lymphocytes were reduced $(p=0.04$ and $p<0.001$, respectively) and (2) CD14+/PD-L1+ monocytes were augmented $(p=0.009$ ) (Table 4; Figure 4). Finally, although not reaching statistical significance, possibly because of the small number of enrolled patients, a clear prevalence of anti-inflammatory monocytes was seen in the HV/LP diet individuals, in whom higher percentages of CD14+/TGF $\beta+$ monocytes were detected $(p=0.09)$ (Table 4).

\section{Correlation between Lachnospiraceae and Anti-Inflammatory Immune Cell}

Lachnospiraceae, the family of bacteria whose abundance was observed to characterize the microbiota of MS diet patients, were recently described to be associated with the preferential generation of an anti-inflammatory milieu. Thus, this family of bacteria facilitates Treg differentiation and stimulates TGF $\beta$ and IL-10 production by immune cells. To verify whether the Lachnospiraceae abundance seen in the HV/LP diet patients could be linked to a modulation of such cells, we analyzed possible correlations between Lachnospiraceae abundance and immune parameters. Results showed in HV/LP diet alone the presence of significantly positive correlations between Lachnospiraceae and both CD14+/IL-10+ and CD14+/TGF $\beta+$ monocytes ( $R_{\mathrm{Sp}}=0.77, p=0.008$ and $R_{\mathrm{Sp}}=0.73, p=0.01$; respectively), as well as between Lachnospiraceae and CD4+/CD25+/FoxP3+ T lymphocytes $\left(R_{\mathrm{Sp}}=0.68, p=0.02\right)$. 


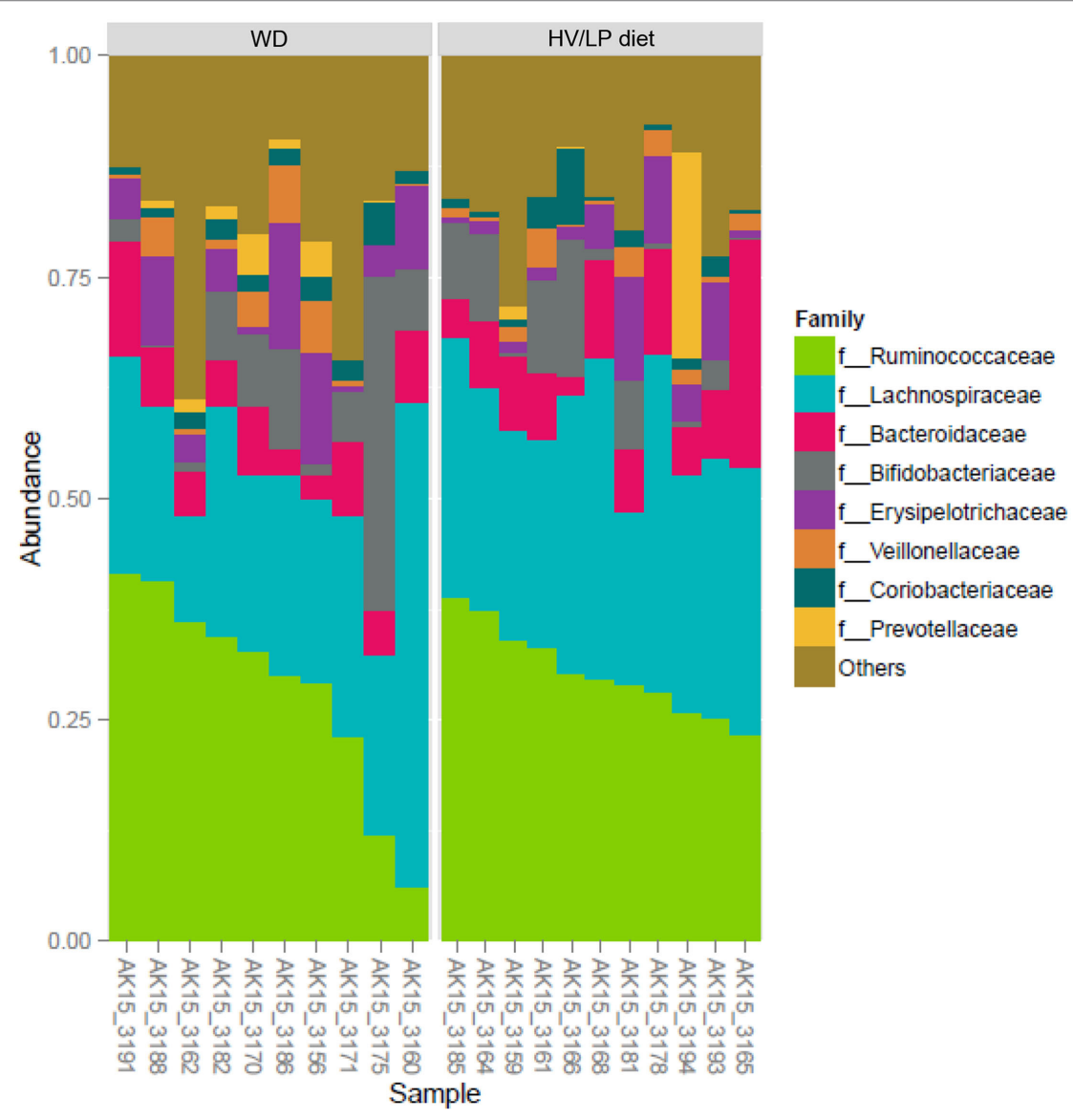

FIGURE 2 | Ruminococcaceae ( 30\%) and Lachnospiraceae ( 27\%) were the most abundant Families, both belong to Firmicutes phylum. Plot showing the most abundant taxa at the Family level in multiple sclerosis patients who were following either a Western Diet (WD) or a high-vegetable/low-protein (HV/LP) diet. Stool samples were collected at enrollment, i.e., after at least 1 year of either WD or HV/LP diet.

Lachnospiraceae were negatively correlated with both $\mathrm{CD} 14+/$ IL- $10+$ and CD $14+/ \mathrm{TGF} \beta+$ monocytes $\left(R_{\mathrm{Sp}}=-0.09, p=0.8\right.$ and $R_{\mathrm{Sp}}=-0.48, p=0.1$, respectively) and positively correlated with CD4+/CD25+/FoxP3+ T lymphocytes $\left(R_{\mathrm{Sp}}=0.02, p=0.9\right)$ in WD; none of these correlations was statistically significant in this group of patients.

\section{Modulation of Disease Activity}

Clinical parameters were evaluated during the 12 months follow-up period as well as at the end of the protocol; interesting differences emerged. Thus, whereas the EDSS score improved in the HV/LP diet patients, this parameter declined in WD patients, with a significant difference being observed between the two groups $(p=0.001)$. Notably, the overall number of disease relapses during the 12 months follow-up period was unmodified in the HV/LP diet patients, but increased significantly in the WD patients (vs. enrollment $p=0.04$ ). Clinical relapses were observed during the 12 months follow-up period in $9 / 10 \mathrm{WD}$ patients but only in $3 / 10$ of the HV/LP diet patients $(p=0.0005)$; the difference between the two groups in relapse rate at the 12 months follow-up visit was statistically significant $(p=0.03)$. These results are shown in Table 5 .

\section{DISCUSSION}

Diet plays an essential role in shaping the composition of the gut microbiome (35), and the gut microbiota modulates the status of the immune response (36). In MS, in particular, changes in the composition of the microbiota were suggested to influence disease activity, and in the EAE murine model of MS tampering with microbiota can trigger or prevent disease development. To better define whether in MS different dietary regimens can modify the microbiota, if this results in a modulation of immune profiles, and, ultimately, whether diet-associated changes in the composition of the microbiota influence disease activity, we analyzed these parameters in two groups of MS patients who were following different diets. In particular, we compared microbiota composition and immune profiles in MS patients that had followed either a HV/LP or a WD diet for at least 1 year; clinical parameters were analyzed in these same individuals during a 12 months follow-up period. Results of this pilot study show that a skewing of the composition of the microbiota characterized by the abundance of Lachnospiraceae family, a decrease of IL-17-producing T CD4+ lymphocytes and $\mathrm{PD}-1$ expressing $\mathrm{T}$ CD4+ lymphocytes, and an increase of 


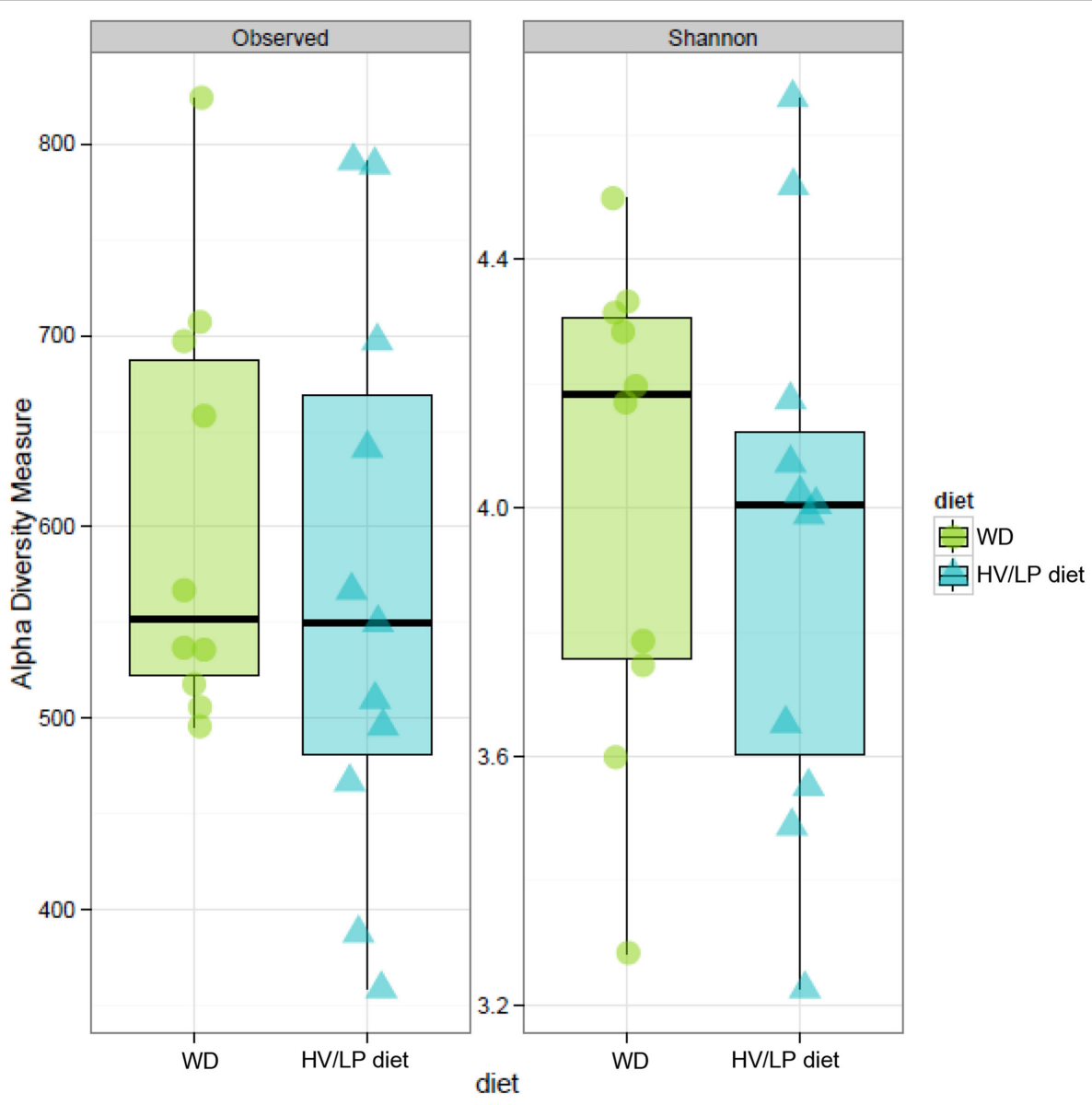

FIGURE 3 | Estimates of alpha-diversity no shown significant difference. Observed diversity (left panel) represents the number of operation taxonomic unit (OTU) present in each sample, while Shannon diversity index (right panel) takes in account of richness and evenness of OTUs within a sample. Stool samples were collected at enrollment, i.e., after at least 1 year of either Western Diet or high-vegetable/low-protein diet.

TABLE 4 | Immune parameters in patients with a diagnosis of multiple sclerosis who were following either a Western Diet (WD) or a high-vegetable/low-protein (HV/LP) diet.

\begin{tabular}{lccc}
\hline & WD & HV/LP diet & p-Value \\
\hline CD4+CD25+FOXP3+ & $2.6(2.0-3.0)$ & $3.1(2.3-3.6)$ & 0.5 \\
CD4+TIM-3+ & $0.8(0.6-0.9)$ & $1.0(0.7-1.4)$ & 0.3 \\
CD4+GAL-9+ & $1.4(0.7-1.7)$ & $1.2(0.6-2.0)$ & 0.8 \\
CD4+BAT3+ & $0.3(0.2-0.3)$ & $0.4(0.3-0.4)$ & 0.2 \\
CD4+PD-1+ & $0.4(0.4-0.4)$ & $0.2(0.1-0.3)$ & $\mathbf{0 . 0 0 0 4}$ \\
CD4+NFATC+ & $0.2(0.1-0.3)$ & $0.1(0.1-0.1)$ & 0.07 \\
CD4+NFkB+ & $0.2(0.2-0.4)$ & $0.2(0.1-0.3)$ & 0.6 \\
CD4+GATA-3+ & $0.2(0.1-0.4)$ & $0.3(0.2-0.4)$ & 0.4 \\
CD4+ROR + & $0.2(0.1-0.3)$ & $0.2(0.2-0.3)$ & 0.5 \\
CD4+IL-10+ & $0.2(0.1-0.2)$ & $0.1(0.1-0.2)$ & 0.1 \\
CD4+BDNF+ & $0.2(0.1-0.2)$ & $0.3(0.2-0.3)$ & 0.2 \\
CD4+IL-25+ & $0.1(0.0-0.2)$ & $0.1(0.0-0.2)$ & 0.9 \\
CD4+IL-17+ & $0.6(0.5-0.7)$ & $0.2(0.1-0.5)$ & $\mathbf{0 . 0 2}$ \\
CD4+IFN + & $0.5(0.0-1.1)$ & $0.4(0.0-1.2)$ & 0.7 \\
CD14+IL-10+ & $0.5(0.3-0.8)$ & $0.9(0.4-1.3)$ & 0.6 \\
CD14+TGF $\beta+$ & $0.9(0.7-1.2)$ & $1.6(0.9-1.9)$ & 0.09 \\
CD14+PD-L1+ & $1.8(1.4-3.2)$ & $5.5(3.9-9.8)$ & $\mathbf{0 . 0 0 9}$
\end{tabular}

Results obtained at enrollment (i.e., after 1 year of either WD or HV/LP diet) are shown. Data are reported as medians and interquartile range. Statistical significances are presented ( $p<0.05)$.

The bold values are statistically significant $p$ values.
PD-L1 expressing monocytes was observed in those individuals following a HV/LP diet. In these same patients, positive correlations between Lachnospiraceae and anti-inflammatory IL-10and TGF $\beta$-producing CD14+ monocytes, as well as between Lachnospiraceae and CD4+/CD25+/FoxP3+ Treg lymphocytes were also observed. Notably, a significant reduction of the EDSS score and of the relapse rate was observed during follow-up in the HV/LP diet group alone.

Different dietary regimens have convincingly been shown to influence the composition of the intestinal microbiota $(35,37$, 38) and are suggested to modulate the clinical phenotype of a number of inflammatory and non-inflammatory conditions (39). Thus, whereas the microbiota was demonstrated to be different when breastfed and formula-fed neonates were compared, in adults dietary changes result in modifications of the gut microbiota. Recent results, in particular, indicated that a diet based on a high consumption of vegetables leads to an increase in the population of Firmicutes (Roseburia, Ruminococcus bromii, and Eubacterium rectale), whereas a primarily meat-based diet results in an increase in the abundance of bile-tolerant microbes (Alistipes, Bilophila, and Bacteroides) (35). We observed that the use of a HV/LP diet in MS patients was linked to an 

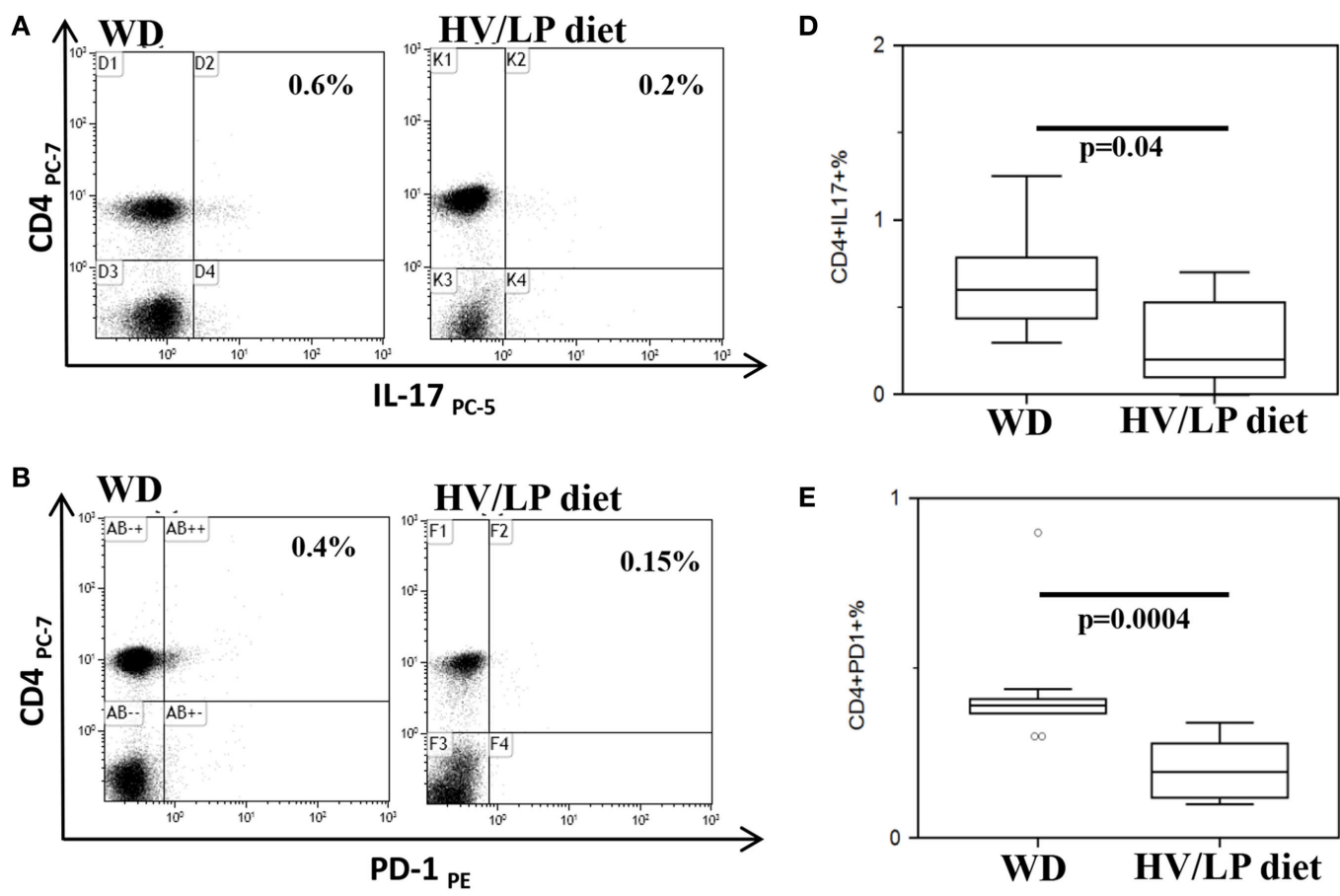

$\mathbf{E}$

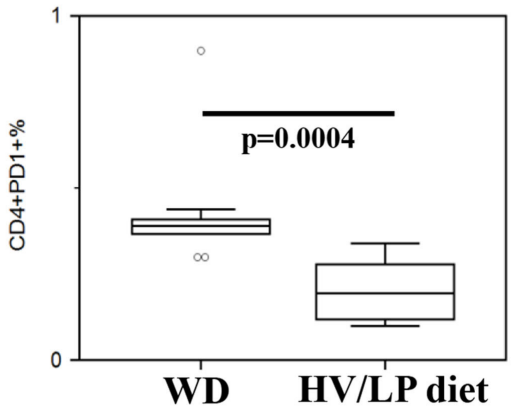

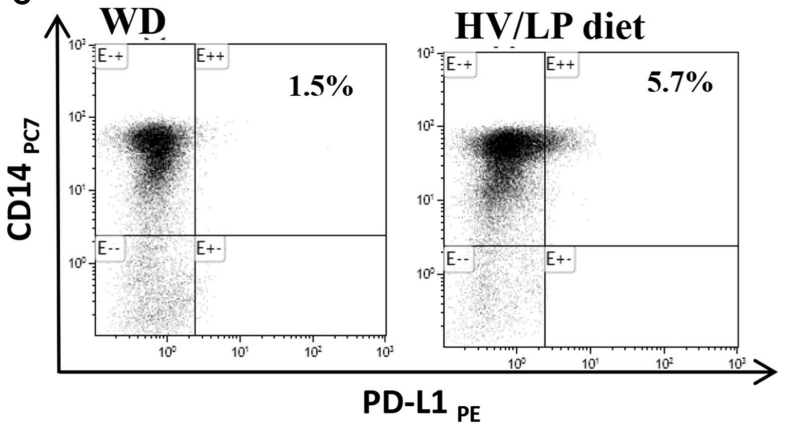

$\mathbf{F}$

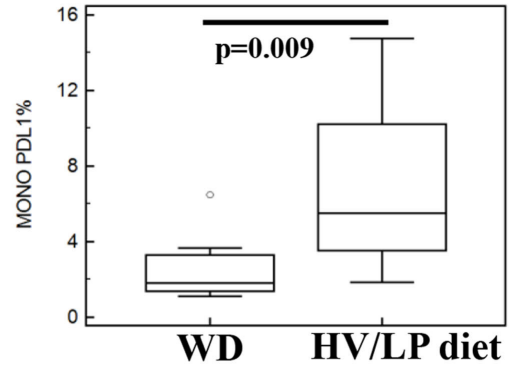

FIGURE 4 | CD4+//L-17+ and CD4+/PD-1+ T lymphocytes are decreased and CD14+/PD-L1+ cells are increased in multiple sclerosis (MS) patients following a high-vegetable/low-protein (HV/LP) diet. IL-17+/CD4+ T lymphocytes (A); PD-1+/CD4+T lymphocytes (B); and PD-L1+/CD14+ cells (C). Representative results obtained in unstimulated peripheral blood mononuclear cell of MS patients who were following either a Western Diet (WD) or a HV/LP diet are shown. Top right corners show the percentage of CD4+/IL-17+, CD4+/PD-1+ T cells and of CD14+/PD-L1+ cells. Summary results are shown in (D-F). The boxes stretch from the 25th to the 75th percentile; the lines across the boxes indicate the median values; the lines stretching from the boxes indicate extreme values. Statistical significance is shown. Blood samples were collected at enrollment, i.e., at least after 1 year of either WD or either HV/LP diet.

abundance of Lachnospiraceae bacteria in the gut microbiota. The Lachnospiraceae family belongs to the Firmicutes phylum, which are butyrate producers. This observation is important from an immunologic viewpoint, as butyrate is endowed with the ability to stimulate Treg activity and differentiation and to induce the generation of anti-inflammatory cytokines, including IL-10, by Treg cells $(27,40)$. Notably, data herein indicate that the abundance in Lachnospiraceae seen in the HV/LP diet MS patients was significantly correlated with increased percentages of peripheral Treg and of IL-10 and TGF $\beta$-producing monocytes. In animal models, butyrate-producing bacteria were also shown to restore the integrity of the intestinal as well as of the BBB (41, 42 ), possibly reducing the translocation of peripheral blood inflammatory cells across the BBB.
We have previously shown that, whereas CD4+/Th17+ and CD4+/PD-1+ T lymphocytes are increased in MS compared to $\mathrm{HC}$ (43), CD14+/PD-L1+ monocytes prevail during disease remission (44); this is the immune profile we observed in MS patients undergoing a HV/LP diet. Taken together, these results could explain the attenuation of disease activity observed in these individuals during follow-up. Previous analyses of the microbiota composition in MS patients showed that Methanobrevibacter, bacteria that have been associated with inflammatory and autoimmune diseases (45), are increased in untreated MS (28) and this leads to a shorter relapse time (46). An increase of Akkermansia was also demonstrated in MS untreated patients $(28,47)$, in whom butyrate-producing Faecalibacterium, Lachnospiraceae, as well as Ruminococcaceae, Bacteroides fragilis, and Butyricimonas 
TABLE 5 | Expanded Disability Status Scale (EDSS) scores, relapse rates, and number of patients in whom disease relapses were observed.

\begin{tabular}{|c|c|c|c|}
\hline & WD & HV/LP diet & $P$-Value \\
\hline EDSS (range) at baseline & $2.0(1.6-2.9)$ & $1.8(1.3-2.0)$ & 0.44 \\
\hline $\begin{array}{l}\text { EDSS (range) at the end } \\
\text { of the follow-up period }\end{array}$ & $2.5(2.1-3.0)$ & $1.0(1.0-1.0)$ & 0.001 \\
\hline $\begin{array}{l}\text { Relapse rate (relapse number/ } \\
\text { disease years) at baseline }\end{array}$ & $0.3(0.3-0.8)$ & $1.0(0.0-1.0)$ & 0.42 \\
\hline $\begin{array}{l}\text { Relapse rate (relapse number/ } \\
\text { disease years) during the } 12 \text { months } \\
\text { follow-up period }\end{array}$ & $1.0(1.0-1.0)$ & $0.0(0.0-1.0)$ & 0.03 \\
\hline EDSS baseline vs. follow-up & 0.31 & 0.06 & \\
\hline \multirow[t]{2}{*}{ Relapse rate baseline vs. follow-up } & 0.04 & 0.6 & \\
\hline & WD & HV/LP diet & $P$-Value \\
\hline $\begin{array}{l}\text { Patients in whom disease } \\
\text { relapses were observed during } \\
\text { the } 12 \text { months follow-up period }\end{array}$ & $9 / 10$ & $3 / 10$ & 0.0005 \\
\hline
\end{tabular}

Two groups of multiple sclerosis patients following either a Western Diet (WD) or a highvegetable/low-protein (HV/LP) diet were analyzed. Results obtained at enrollment (i.e., after 1 year of either WD or HVILP diet) as well as during a 12 months follow-up period are presented. Medians, interquartile ranges and statistical significances are shown $(p<0.05)$.

The bold values are statistically significant $p$ values.

were reduced $(28,47,48)$ Finally, Prevotella, a genus including many butyrate producers (49), was less abundant in MS untreated patients $(4,50)$, but an increase of this bacteria as well as of Sutterella, a species that drives anti-inflammatory response, was demonstrated in MS after treatment $(28,51)$. Results of this pilot study show a higher prevalence of Lachnospiraceae in MS patients following a HV/LP diet and are in accordance with previous data showing an increase of butyrate-producing bacteria in MS individuals after immunomodulatory treatment $(28,51)$. These data support the possibility that diet could be possibly be used as a tool to modulate the immune system in anti-inflammatory way as a consequence of changes in the gut microbiota $(52,53$, 54). Notably, our results also showed that phylum Euryarchaeota was increased in WD patients alone. This finding could be of interest as this phylum was recently suggested to associate with shorter time to disease relapse in pediatric MS patients (55). Intriguingly, and underlining once again the intricacies of the interactions between the different components of the microbiota, a negative correlation between taxa belonging to family Lachnospiraceae, and Methanomassiliicoccales, which belong to phylum Euryarchaeota, was described (56). This is immunologically important as archaea of the Euryarchaeota phylum

\section{REFERENCES}

1. Wang Y, Kasper LH. The role of microbiome in central nervous system disorders. Brain Behav Immun (2014) 38:1-12. doi:10.1016/j.bbi.2013.12.015

2. Bhargava P, Mowry EM. Gut microbiome and multiple sclerosis. Curr Neurol Neurosci Rep (2014) 14(10):492. doi:10.1007/s11910-014-0492-2

3. Ochoa-Reparaz J, Mielcarz DW, Begum-Haque S, Kasper LH. Gut, bugs, and brain: role of commensal bacteria in the control of central nervous system disease. Ann Neurol (2011) 69:240-7. doi:10.1002/ana.22344

4. Miyake S, Kim S, Suda W, Oshima K, Nakamura M, Matsuoka T, et al. Dysbiosis in the gut microbiota of patients with multiple sclerosis, with a striking depletion of species belonging to Clostridia XIVa and IV clusters. PLoS One (2015) 10(9):e0137429. doi:10.1371/journal.pone.0137429 were suggested to interact with dendritic cells, resulting in the production of proinflammatory cytokines (57).

It is important to notice that this is a classic pilot study. Thus, because baseline samples were not collected, it is not possible to definitely state that the microbiota, immunologic, and clinical changes described herein are the direct consequence of the dietary regimens. These caveats notwithstanding, our data could be seen as supportive of the concept that diet-associated modifications of the composition of the microbiota modulate the immune response, and, in turn, this has an important impact on disease activity. It will be important to replicate these results in ampler cohorts of MS patients and to expand these observations in inflammatory diseases other that MS.

\section{ETHICS STATEMENT}

All patients gave informed consent according to a protocol approved by the local ethics committee of the Don Gnocchi Foundation.

\section{AUTHOR CONTRIBUTIONS}

MS designed and performed the study and drafted the manuscript; LM designed the study and collected clinical data; FM organized patients enrollment and collected blood samples; DC selected patient groups and collected clinical data; GF organized and wrote the microbiota data; FP, FL, and IM performed immunological experiments and analyzed the results; VR conducted and analyzed nutritional interviews; MC coordinated the study and edited the manuscript. All authors reviewed and approved the final manuscript.

\section{FUNDING}

This research was supported by a generous contribution by the MS patients that are part of the "Microbiota and Multiple Sclerosis Research" and by Italian Ministry of Health 2015-2016.

\section{SUPPLEMENTARY MATERIAL}

The Supplementary Material for this article can be found online at http://www.frontiersin.org/article/10.3389/fimmu.2017.01391/ full\#supplementary-material.

5. Ochoa-Reparaz J, Mielcarz DW, Ditrio LE, Burroughs AR, Foureau DM, Haque-Begum S, et al. Role of gut commensal microflora in the development of experimental autoimmune encephalomyelitis. JImmunol (2009) 183:6041-50. doi:10.4049/jimmunol.0900747

6. Ochoa-Reparaz J, Mielcarz DW, Haque-Begum S, Kasper LH. Induction of a regulatory B cell population in experimental allergic encephalomyelitis by alteration of the gut commensal microflora. Gut Microbes (2010) 1:103-8. doi:10.4161/gmic.1.2.11515

7. Lee YK, Menezes JS, Umesaki Y, Mazmanian SK. Proinflammatory T-cell responses to gut microbiota promote experimental autoimmune encephalomyelitis. Proc Natl Acad Sci U S A (2011) 108(Suppl):4615-22. doi:10.1073/pnas.1000082107

8. Ezendam J, de Klerk A, Gremmer ER, van Loveren H. Effects of Bifidobacterium animalis administered during lactation on allergic and 
autoimmune responses in rodents. Clin Exp Immunol (2008) 154(3):424-31. doi:10.1111/j.1365-2249.2008.03788.x

9. Maassen CB, Claassen E. Strain-dependent effects of probiotic lactobacilli on EAE autoimmunity. Vaccine (2008) 26(17):2056-7. doi:10.1016/j.vaccine. 2008.02.035

10. Kobayashi T, Kato I, Nanno M, Shida K, Shibuya K, Matsuoka Y, et al. Oral administration of probiotic bacteria, Lactobacillus casei and Bifidobacterium breve, does not exacerbate neurological symptoms in experimental autoimmune encephalomyelitis. Immunopharmacol Immunotoxicol (2010) 32(1):116-24. doi:10.3109/08923970903200716

11. Kobayashi T, Suzuki T, Kaji R, Serata M, Nagata T, Ando M, et al. Probiotic upregulation of peripheral IL-17 responses does not exacerbate neurological symptoms in experimental autoimmune encephalomyelitis mouse models. Immunopharmacol Immunotoxicol (2012) 34(3):423-33. doi :10.3109/08923973.2010.617755

12. Lavasani S, Dzhambazov B, Nouri M, Fåk F, Buske S, Molin G, et al. A novel probiotic mixture exerts a therapeutic effect on experimental autoimmune encephalomyelitis mediated by IL-10 producing regulatory T cells. PLoS One (2010) 5(2):e9009. doi:10.1371/journal.pone.0009009

13. Ochoa-Reparaz J, Mielcarz DW, Ditrio LE, Burroughs AR, Begum-Haque S, Dasgupta $S$, et al. Central nervous system demyelinating disease protection by the human commensal Bacteroides fragilis depends on polysaccharide A expression. JImmunol (2010) 185:4101-8. doi:10.4049/jimmunol. 1001443

14. Ochoa-Reparaz J, Mielcarz DW, Wang Y, Begum-Haque S, Dasgupta S, Kasper DL, et al. A polysaccharide from the human commensal Bacteroides fragilis protects against CNS demyelinating disease. Mucosal Immunol (2010) 3:487-95. doi:10.1038/mi.2010.29

15. Takata K, Kinoshita M, Okuno T, Moriya M, Kohda T, Honorat JA, et al. The lactic acid bacterium Pediococcus acidilactici suppresses autoimmune encephalomyelitis by inducing IL-10-producing regulatory T cells. PLoS One (2011) 6(11):e27644. doi:10.1371/journal.pone.0027644

16. Kwon HK, Kim GC, Kim Y, Hwang W, Jash A, Sahoo A, et al. Amelioration of experimental autoimmune encephalomyelitis by probiotic mixture is mediated by a shift in T helper cell immune response. Clin Immunol (2013) 146(3):217-27. doi:10.1016/j.clim.2013.01.001

17. Ochoa-Repáraz J, Riccardi C, Rynda A, Jun S, Callis G, Pascual DW. Regulatory $\mathrm{T}$ cell vaccination without autoantigen protects against experimental autoimmune encephalomyelitis. J Immunol (2007) 178(3):1791-9. doi:10.4049/jimmunol.178.3.1791

18. Ochoa-Repáraz J, Rynda A, Ascón MA, Yang X, Kochetkova I, Riccardi C, et al. IL-13 production by regulatory $\mathrm{T}$ cells protects against experimental autoimmune encephalomyelitis independently of autoantigen. J Immunol (2008) 181(2):954-68. doi:10.4049/jimmunol.181.2.954

19. Rezende RM, Oliveira RP, Medeiros SR, Gomes-Santos AC, Alves AC, Loli FG, et al. Hsp65-producing Lactococcus lactis prevents experimental autoimmune encephalomyelitis in mice by inducing CD4+LAP+ regulatory T cells. J Autoimmun (2013) 40:45-57. doi:10.1016/j.jaut.2012. 07.012

20. Piccio L, Stark JL, Cross AH. Chronic calorie restriction attenuates experimental autoimmune encephalomyelitis. JLeukoc Biol (2008) 84:940-8. doi:10.1189/jlb.0208133

21. Kleinewietfeld M, Manzel A, Titze J, Kvakan H, Yosef N, Linker RA, et al. Sodium chloride drives autoimmune disease by the induction of pathogenic TH17 cells. Nature (2013) 496:518-22. doi:10.1038/nature11868

22. Rumah KR, Linden J, Fischetti VA, Vartanian T. Isolation of Clostridium perfringens type $\mathrm{B}$ in an individual at first clinical presentation of multiple sclerosis provides clues for environmental triggers of the disease. PLoS One (2013) 8:e76359. doi:10.1371/journal.pone.0076359

23. Mete A, Garcia J, Ortega J, Lane M, Scholes S, Uzal FA. Brain lesions associated with Clostridium perfringens type D epsilon toxin in a Holstein heifer calf. Vet Pathol (2013) 50:765-8. doi:10.1177/0300985813476058

24. Dorca-Arévalo J, Soler-Jover A, Gibert M, Popoff MR, Martín-Satué M, Blasi J. Binding of epsilon-toxin from Clostridium perfringens in the nervous system. Vet Microbiol (2008) 131:14-25. doi:10.1016/j.vetmic.2008. 02.015

25. Lonchamp E, Dupont JL, Wioland L, Courjaret R, Mbebi-Liegeois C, Jover E, et al. Clostridium perfringens epsilon toxin targets granule cells in the mouse cerebellum and stimulates glutamate release. PLoS One (2010) 5:e13046. doi:10.1371/journal.pone.0013046

26. Finnie JW, Blumbergs PC, Manavis J. Neuronal damage produced in rat brains by Clostridium perfringens type D epsilon toxin. J Comp Pathol (1999) 120:415-20. doi:10.1053/jcpa.1998.0289

27. Atarashi K, Tanoue T, Oshima K, Suda W, Nagano Y, Nishikawa H, et al. Treg induction by a rationally selected mixture of Clostridia strains from the human microbiota. Nature (2013) 500:232-6. doi:10.1038/nature12331

28. Jangi S, Gandhi R, Cox LM, Li N, von Glehn F, Yan R, et al. Alterations of the human gut microbiome in multiple sclerosis. Nat Commun (2016) 7:12015. doi:10.1038/ncomms12015

29. Glenn JD, Mowry EM. Emerging concepts on the gut microbiome and multiple sclerosis. J Interferon Cytokine Res (2016) 36(6):347-57. doi:10.1089/ jir.2015.0177

30. Colon MA, Bird AR. The impact of diet and lifestyle on gut microbiota and human health. Nutrients (2014) 7(1):17-44. doi:10.3390/nu7010017

31. Slavin J. Fiber and prebiotics: mechanisms and health benefits. Nutrients (2013) 5:1417-35. doi:10.3390/nu5041417

32. Hu FB, Rimm EB, Stampfer MJ, Ascherio A, Spiegelman D, Willett WC. Prospective study of major dietary patterns and risk of coronary heart disease in men. Am J Clin Nutr (2000) 72(4):912-21.

33. Bray JR, Curtis JT. An ordination of upland forest communities of southern Wisconsin. Ecol Monogr (1957) 27:325-49. doi:10.2307/1942268

34. Jaccard P. The distribution of the flora in the alpine zone. New Phytol (1912) 11:37-50. doi:10.1111/j.1469-8137.1912.tb05611.x

35. David LA, Maurice CF, Carmody RN, Gootenberg DB, Button JE, Wolfe BE, et al. Diet rapidly and reproducibly alters the human gut microbiome. Nature (2014) 505(7484):559-63. doi:10.1038/nature12820

36. Petersen C, Round JL. Defining dysbiosis and its influence on host immunity and disease. Cell Microbiol (2014) 16(7):1024-33. doi:10.1111/cmi.12308

37. Turnbaugh PJ, Ridaura VK, Faith JJ, Rey FE, Knight R, Gordon JI. The effect of diet on the human gut microbiome: a metagenomic analysis in humanized gnotobiotic mice. Sci Transl Med (2009) 1(6):6ra14. doi:10.1126/ scitranslmed.3000322

38. De Filippo C, Cavalieri D, Di Paola M, Ramazzotti M, Poullet JB, Massart S. Impact of diet in shaping gut microbiota revealed by a comparative study in children from Europe and rural Africa. Proc Natl Acad Sci U S A (2010) 107(33):14691-6. doi:10.1073/pnas.1005963107

39. Maslowski KM, Mackay CR. Diet, gut microbiota and immune responses. Nat Immunol (2011) 12:5-9. doi:10.1038/ni0111-5

40. Sun M, He C, Cong Y, Liu Z. Regulatory immune cells in regulation of intestinal inflammatory response to microbiota. Mucosal Immunol (2015) 8(5):969-78. doi:10.1038/mi.2015.49

41. Braniste V, Al-Asmakh M, Kowal C, Anuar F, Abbaspour A, Tóth M, et al. The gut microbiota influences blood-brain barrier permeability in mice. Sci Transl Med (2014) 6:263ra158. doi:10.1126/scitranslmed.3009759

42. Ochoa-Repáraz J, Kasper LH. The influence of gut-derived CD39 regulatory T cells in CNS demyelinating disease. Transl Res (2017) 179:126-38. doi:10.1016/j.trsl.2016.07.016

43. Saresella M, Tortorella P, Marventano I, Al-Daghri N, Piancone F, Gatti A, et al. TH17-driven inflammation is present in all clinical forms of multiple sclerosis; disease quiescence is associated with GATA3-expressing cells. Eur J Inflamm (2013) 11(1):223-35. doi:10.1177/1721727X13 01100121

44. Trabattoni D, Saresella M, Pacei M, Marventano I, Mendozzi L, Rovaris M, et al. Costimulatory pathways in multiple sclerosis: distinctive expression of PD-1 and PD-L1 in patients with different patterns of disease. J Immunol (2009) 183(8):4984-93. doi:10.4049/jimmunol.0901038

45. Verma R, Verma AK, Ahuja V, Paul J. Real-time analysis of mucosal flora in patients with inflammatory bowel disease in India. J Clin Microbiol (2010) 48:4279-82. doi:10.1128/JCM.01360-10

46. Tremlett H, Fadrosh DW, Faruqi AA, Hart J, Roalstad S, Graves J, et al. Gut microbiota composition and relapse risk in pediatric MS: a pilot study. J Neurol Sci (2016) 363:153-7. doi:10.1016/j.jns.2016.02.042

47. Cantarel BL, Waubant E, Chehoud C, Kuczynski J, DeSantis TZ, Warrington J, et al. Gut microbiota in multiple sclerosis: possible influence of immunomodulators. J Investig Med (2015) 63(5):729-34. doi:10.1097/ JIM.0000000000000192 
48. Tremlett H, Fadrosh DW, Faruqi AA, Zhu F, Hart J, Roalstad S, et al. Gut microbiota in early pediatric multiple sclerosis: a case-control study. Eur J Neurol (2016) 23:1308-21. doi:10.1111/ene.13026

49. Wu GD, Chen J, Hoffmann C, Bittinger K, Chen Y, Keilbaugh SA, et al. Lewis linking long-term dietary patterns with gut microbial enterotypes. Science (2011) 334(6052):105-8. doi:10.1126/science.1208344

50. Chen J, Chia N, Kalari KR, Yao JZ, Novotna M, Soldan MM, et al. Multiple sclerosis patients have a distinct gut microbiota compared to healthy controls. Sci Rep (2016) 6:28484. doi:10.1038/srep2848

51. Castillo Alvarez F, Perez Matute P, Colina Lizuain S, Erdocia Goni A, Gutiérrez Cecchini C, Gòmez Eguilaz M, et al. Intestinal microbiota in multiple sclerosis: Influence of treatment with interferon $\beta$-1 $\beta$. Proceedings of the 2nd ECTRIMS; 2016, Sept 14-17. London, UK: (2016). Available from: https:// onlinelibrary.ectrims-congress.eu/ectrims/2016/32nd/146290/federico.castillo.lvarez.intestinal.microbiota.in.multiple.sclerosis.influence.html? $\mathrm{f}=\mathrm{m} 3$

52. Haghikia A, Jörg S, Duscha A, Berg J, Manzel A, Waschbisch A, et al. Dietary fatty acids directly impact central nervous system autoimmunity via the small intestine. Immunity (2015) 43(4):817-29. doi:10.1016/j.immuni. 2015.09.007

53. Budhram A, Parvathy S, Kremenchutzky M, Silverman M. Breaking down the gut microbiome composition in multiple sclerosis. Mult Scler (2017) 23(5):628-36. doi:10.1177/1352458516682105

54. Riccio P, Rossano R. Nutrition facts in multiple sclerosis. ASN Neuro (2015) 7. doi:10.1177/1759091414568185
55. Tremlett H, Fadrosh DW, Faruqi AA, Hart J, Roalstad S, Graves J, et al. Gut microbiota composition and relapse risk in pediatric MS: A pilot study. J Neurol Sci (2016) 363:153-7. doi:10.1016/j.jns.2016.02.042

56. Vanderhaeghen S, Lacroix C, Schwab C. Methanogen communities in stools of humans of different age and health status and co-occurrence with bacteria. FEMS Microbiol Lett (2015) 362(13):fnv092. doi:10.1093/femsle/ fnv092

57. Bang C, Weidenbach K, Gutsmann T, Heine H, Schmitz RA. The intestinal archaea Methanosphaera stadtmanae and Methanobrevibacter smithii activate human dendritic cells. PLoS One (2014) 9(6):e99411. doi:10.1371/ journal.pone.0099411

Conflict of Interest Statement: The authors declare that the research was conducted in the absence of any commercial or financial relationships that could be construed as a potential conflict of interest.

Copyright (C) 2017 Saresella, Mendozzi, Rossi, Mazzali, Piancone, LaRosa, Marventano, Caputo, Felis and Clerici. This is an open-access article distributed under the terms of the Creative Commons Attribution License (CC BY). The use, distribution or reproduction in other forums is permitted, provided the original author(s) or licensor are credited and that the original publication in this journal is cited, in accordance with accepted academic practice. No use, distribution or reproduction is permitted which does not comply with these terms. 